\title{
Leader-Follower Containment Control Over Directed Random Graphs
}

\author{
Zhen Kan $^{\mathrm{a}}$, John M. Shea ${ }^{\mathrm{b}}$, Warren E. Dixon ${ }^{\mathrm{a}}$ \\ ${ }^{a}$ Department of Mechanical and Aerospace Engineering, University of Florida, Gainesville, USA \\ ${ }^{\mathrm{b}}$ Department of Electrical and Computer Engineering, University of Florida, Gainesville, USA.
}

\begin{abstract}
The leader-follower consensus problem for multi-agent systems over directed random graphs is investigated. Motivated by the fact that inter-agent communication can be subject to random failure when agents perform tasks in a complex environment, a directed random graph is used to model the random loss of communication between agents, where the connection of the directed edge in the graph is assumed to be probabilistic and evolves according to a two-state Markov Model. In the leaderfollower network, the leaders maintain a constant desired state and the followers update their states by communicating with local neighbors over the random communication network. Based on convex properties and a stochastic version of LaSalle's Invariance Principle, almost sure convergence of the followers' states to the convex hull spanned by the leaders' states is established for the leader-follower random network. A numerical simulation is provided to demonstrate the developed result.
\end{abstract}

\section{Introduction}

Consensus problems that seek to agree upon certain quantities of interest have attracted significant research attention. A comprehensive review of consensus problems is provided in [1] and [2]. To achieve consensus, agents are generally required to exchange information over a communication network as a means to coordinate their behaviors, such as achieving a common heading direction in flocking problems [3,4], agreeing on the group average in distributed sensing [5], or achieving consensus in rendezvous and formation control problems [6,7], to name a few. In most of these applications, consistent information exchange between agents in either an undirected or directed manner is a common assumption to ensure full cooperation among team members. However, when agents operate in a complex environment, the inter-agent communication could be subject to random failure due to either interference or unpredictable environmental disturbance. Since task completion relies on

Email addresses: kanzhen0322@ufl.edu (Zhen Kan), jshea@ece.ufl.edu (John M. Shea), wdixon@ufl.edu (Warren E. Dixon).

1 This research is supported in part by NSF award numbers 1161260, 1217908, and a contract with the AFRL Mathematical Modeling and Optimization Institute. Any opinions, findings and conclusions or recommendations expressed in this material are those of the authors and do not necessarily reflect the views of the sponsoring agency. communication and interaction among agents, achieving consensus over such a stochastic communication network can be challenging.

Leader-follower containment control is a particular class of consensus problems, in which the networked multiagent system consists of leader agents and follower agents. Generally, the leaders are a small subset of the agents, which are informed of the global task objectives, while the followers act under the influence of both neighboring agents and the leaders through local interactions. A main objective in leader-follower containment control is to drive all followers' states to a desired destination determined by the leaders' states. Hybrid control schemes are developed in [8] to drive the dynamic follower agents into a convex polytope spanned by the stationary leader agents, where the local interaction among agents is modeled as an undirected graph. The work of [8] is then extended to multiple stationary and dynamic leaders under a directed interaction graph in [9-11]. Containment control for a leader follower network under a switching graph is studied in [12] and [13]. In [14], containment control is applied to a social network to regulate the emotional states of individuals to a desired end. For networked Lagrangian systems with parametric uncertainties, distributed containment control is developed in [15]. In the aforementioned works, a deterministic dynamic system is considered, where dynamic agents communicate and coordinate with other agents over an undirected or directed deterministic communication net- 
work. Mean-square containment control of a multi-agent system with communication noise is considered in [16]. Since the results developed in [8-16] may not be applicable to stochastic communication networks where the existing communication links experience random loss, an extension of the classical containment control from the deterministic network to the stochastic network is desirable.

Building on graph theory and probability theory, several consensus results have been developed for random graphs. One of the earliest consensus results over an undirected random network is reported in [17], which proves that agreement can be achieved almost surely if the communication links between any pair of agents are activated independently with a common probability. The undirected random graph in [17] is extended to a general class of directed random graphs in [18] and [19]. Necessary and sufficient conditions for consensus are developed in [20] for graphs that are generated by an ergodic and stationary random process. Meansquare-robust consensus over a network with communication noise and random packet loss is considered in the work of [21] and [22]. Stochastic consensus for a multiagent system with communication noise and Markovian switching topologies is investigated in [23]. However, the convergence results reported in [17-23] are only developed for leaderless networks without considering how the leaders can influence the followers to a desired end.

In this paper, the classical leader-follower containment control problem for deterministic systems is extended to a stochastic scenario. The leader-follower network is tasked to drive all followers into a prespecified destination area (i.e., the convex hull spanned by the leaders' states) under the influence of the leaders. Only the leaders are assumed to have the knowledge of the destination. To move toward the specified destination, the followers communicate and update their states with neighboring agents over a communication network. Since wireless communication is subject to random failure due to factors such as fading and packet loss, the inter-agent communication is modeled as a random graph, where each link evolves according to a two-state Markov Model to model the random loss of the existing communication link. In addition, the random communication network is assumed to be directed. Rather than assuming that all edges share a common edge probability and evolve independently with their previous edge connection states as in [17], different edges are allowed to have different transition probabilities in the current work that evolve according to a Markov Model, which can be used to model a large class of real-world networks to reflect the dependence of the current system states on their previous states. Moreover, compared to the works of [17-20], a hierarchical network structure (i.e., leader-follower network) is considered where one-sided influence of leaders is used to affect the desired behaviors of the followers. Almost sure convergence of the followers' states to the convex hull spanned by the leaders' states over a random communication graph is then established via the convex properties in [24] and a stochastic version of LaSalle's Invariance Theorem [25].

\section{Problem Formulation}

A multi-agent system consisting of $n$ agents that communicate over wireless channels is considered. The wireless channels have intermittent connectivity, which cause the connections among the agents to vary with time. The communication graph is modeled as a temporal network, or time-varying graph, $\mathcal{G}(t)=(\mathcal{V}, \mathcal{E}(t))$. The vertices $\mathcal{V}$ represent the agents, which do not vary with time. The edges $\mathcal{E}(t)$ represent the connections among the agents and do vary with time. The flow of information is assumed to be asymmetric, so the edges in $\mathcal{E}(t) \subset \mathcal{V} \times \mathcal{V}$ are directed. Specifically, the directed edge $\left(v_{j}, v_{i}\right) \in \mathcal{E}$ indicates that node $v_{i}$ can receive information from node $v_{j}$, but $v_{j}$ may not necessarily receive information from $v_{i}$. In the directed edge $\left(v_{j}, v_{i}\right), v_{i}$ and $v_{j}$ are referred to as the child node and the parent node, respectively.

\subsection{Directed Random Graph}

Consider first the graph at one particular time, say $t=$ $t_{0}$. Then, suppressing the time dependence, $\mathcal{G}=(\mathcal{V}, \mathcal{E})$ is a directed graph. The graph $\mathcal{G}$ is called a directed random graph if the set of edges $\mathcal{E}$ is randomly determined. Let $\overline{\mathcal{E}} \subset \mathcal{V} \times \mathcal{V}$ be a set of potential directed edges connecting the nodes in $\mathcal{V}$. Each potential edge $\left(v_{j}, v_{i}\right)$ is associated with a weight $w_{i j} \in \mathbb{R}^{+}$, which indicates how node $v_{i}$ evaluates the information collected from $v_{j}$. We assume that the weight $w_{i j}$ for each $\left(v_{j}, v_{i}\right)$ is known initially and there are no self loops, so $\left(v_{i}, v_{i}\right) \notin \overline{\mathcal{E}}, i=1,2$, $\ldots, n$. Associated with each potential edge $\left(v_{j}, v_{i}\right) \in \overline{\mathcal{E}}$, let there be a Bernoulli random variable $\delta_{i j}$. An edge $\left(v_{j}, v_{i}\right) \in \overline{\mathcal{E}}$ will exist in $\mathcal{E}$ if $\delta_{i j}=1$ and will not exist in $\mathcal{E}$ if $\delta_{i j}=0$. It is assumed that, for different edges, the $\left\{\delta_{i j}\right\}$ are statistically independent.

Now, consider the temporal network, $\mathcal{G}(t)$, which consists of a time sequence of directed random graphs in which the edge set varies with $t$. In particular, each edge $(i, j)$ evolves according to a two-state homogeneous Markov process $\delta_{i j}(t)$ for $i, j \in\{1,2, \ldots, n\}$ with stationary state transition probability $p_{i j} \in(0,1]$, which indicates that, at the next time instant $t^{\prime}$, the edge $(i, j)$ will change its state to $\delta_{i j}\left(t^{\prime}\right)=1-\delta_{i j}(t)$ with probability $p_{i j}$ and will remain the previous state $\delta_{i j}\left(t^{\prime}\right)=\delta_{i j}(t)$ with probability $1-p_{i j}$.

Assumption 1 The random processes $\left\{\delta_{i j}(t)\right\}$ do not change infinitely fast, and thus we can choose a sampling time $\Delta_{t}$ such that with arbitrarily high probability, $\delta_{i j}(t)=\delta_{i j}\left(t+t_{0}\right)$ if $0 \leq t_{0}<\Delta_{t}$ for all $i$, $j \in\{1,2, \ldots, n\}$. 
Note that Assumption 1 will be true for any real system. For example, let $T_{0}$ and $T_{1}$ denote the expected dwell times in states 0 and 1 for the Markov process $\delta_{i j}(t)$, respectively. Then, the probability of staying in the same state during an observation period can be made arbitrarily large by selecting an appropriate $\Delta_{t}$. For example, the probability of remaining in state 0 during an interval of length $\Delta_{t}$ is $e^{-\Delta_{t} / T_{0}}$.

We assume that the sequence of random graphs can be discretized in the following way. Let $t_{k}=k \Delta_{t}, k \in \mathbb{Z}^{+}$ be a time sequence, where $\Delta_{t} \in \mathbb{R}^{+}$is a sufficiently small sampling period during which we may assume the edge set is constant over each time interval $\left[t_{k}, t_{k+1}\right)$. Let $\mathcal{G}(k)$ denote the random graph $\mathcal{G}(t)$ at $t=t_{k}$. Note that $\mathcal{G}(k)$ is drawn from a finite sample space, which we denote by $\overline{\mathcal{G}}=\left\{\mathcal{G}_{1}, \ldots, \mathcal{G}_{M}\right\}$, and $|\overline{\mathcal{G}}| \leq 2^{|\overline{\mathcal{E}}|}$, which is determined by the power set of $\overline{\mathcal{E}}$. In a directed graph, a directed path from node $v_{1}$ to node $v_{k}$ is a sequence of edges $\left(v_{1}, v_{2}\right),\left(v_{2}, v_{3}\right), \cdots,\left(v_{i}, v_{k}\right)$. If a directed graph contains a directed spanning tree, every node has exactly one parent node except for one node, called the root, and the root has directed paths to every other node. A directed graph is called strongly connected if there exists a directed path from every node to every other node in the graph. The weighted adjacency matrix $A(k)=$ $\left[a_{i j}(k)\right] \in \mathbb{R}^{n \times n}$ of the directed random graph $\mathcal{G}(k)$ is

$$
a_{i j}(k)=w_{i j} \delta_{i j}(k),
$$

where $w_{i j} \in \mathbb{R}^{+}$is the edge weight. The Laplacian matrix $L(k)$ is then defined as $L(k)=D(k)-A(k)$, where $D(k) \triangleq\left[d_{i j}(k)\right] \in \mathbb{R}^{N \times N}$ is a diagonal matrix with the diagonal entry $d_{i i}(k)=\sum_{j=1}^{N} a_{i j}(k)$, and the offdiagonal entry $d_{i j}(k)=0$ for $\forall i \neq j$. Although $A$ is a random matrix, the Laplacian matrix $L$ is always a zero row sum matrix by its construction, which indicates that 0 is always an eigenvalue of $L$ with the corresponding right eigenvector of $\mathbf{1}=[1, \ldots, 1]^{T} \in \mathbb{R}^{n}$.

\subsection{Leader-Follower Network over Directed Random Graphs}

Consider a multi-agent system composed of $n$ agents that interact over a temporal network $\mathcal{G}(t)=(\mathcal{V}, \mathcal{E}(t))$, where, at each time, the graph $\mathcal{G}(t)$ is a directed random graph, as described in Section 2.1. Suppose that the $n$ agents in $\mathcal{V}$ are partitioned into sets of $\mathcal{V}_{L}$ with $n_{L} \in \mathbb{Z}^{+}$ leaders and $\mathcal{V}_{F}$ with $n_{f} \in \mathbb{Z}^{+}$followers. Let $x_{i}(k) \in$ $\mathbb{R}^{d}$ denote the states (e.g., the Euclidean position) of agent $v_{i} \in \mathcal{V}$ at the time instant $t_{k}$. Only the leaders are assumed to have the immutable and desired states that specifies the locations of the destination area where all agents are required to meet. The followers can only communicate with neighboring agents and update their states over the random communication graph $\mathcal{G}(t)$. Some directed graphs $\mathcal{G}_{i} \in \overline{\mathcal{G}}$ can even be disconnected. Let
$\mathcal{G}_{T}=(\mathcal{V}, \overline{\mathcal{E}})$ be the graph that contains all possible edges for the graphs in $\overline{\mathcal{G}}$. To ensure the followers can be influenced by the leaders to the desired destination area over the directed random network, we introduce the following assumptions.

Assumption 2 It is assumed that $\mathcal{G}_{T} \in \overline{\mathcal{G}}$ and $\operatorname{Pr}\left(\bigcap_{j=k}^{k+n_{f}-1} \mathcal{G}(j)=\mathcal{G}_{T}\right)>0$ for all $k$.

Note that Assumption 2 will be true in many real systems and practical models. For instance, it will be true for the Markov Model described after Assumption 1 if the Markov processes for the different edges are statistically independent. Such independent Markov Models can be used to model the effects of channel outages caused by multipath propagation. However, independence is not required and may not be present in some scenarios. For instance, if communication across the network is randomly blocked by a time-varying jammer, then the links may be completely dependent and yet Assumption 2 still holds.

Assumption 3 The graph $\mathcal{G}_{T} \in \overline{\mathcal{G}}$ has a directed spanning tree, where, for each follower $v_{i} \in \mathcal{V}_{F}$, there exists at least one leader that has a directed path to the follower $v_{i}$.

Assumption 3 implies that the set of leaders act as the roots of the directed spanning tree in $\mathcal{G}_{T}$, which indicates the leaders have an influence directly or indirectly on all followers through a series of directed paths in the network. Different from the objective in [17] and [19], where every node has a probability to connect with every other node in the network, this work relaxes the constraint of having a strongly connected graph.

\subsection{Objective}

Definition 1 [24, Ch. 2] For a set of points $z \triangleq$ $\left\{z_{1}, \cdots, z_{n}\right\}$, the convex hull $\mathrm{Co}(z)$ is defined as the minimal set containing all points in $z$, satisfying

$$
\operatorname{Co}(z) \triangleq\left\{\sum_{i=1}^{n} \alpha_{i} z_{i} \mid z_{i} \in z, \alpha_{i}>0, \sum_{i=1}^{n} \alpha_{i}=1\right\} .
$$

Definition 2 [26, Ch. 4] A state $z(k)$ approaches a set $\mathcal{M}$ as $t_{k}$ goes to infinity (i.e., $z(k) \rightarrow \mathcal{M}$ as $t_{k} \rightarrow$ $\infty)$, if for each $\varepsilon>0$ there exists a $T>0$ such that $\operatorname{dist}(z(k), \mathcal{M})<\varepsilon$ for $t_{k}>T$, where dist $(z(k), \mathcal{M})$ denotes the distance from a point $z(k)$ to a set $M$. More precisely,

$$
\operatorname{dist}(z(k), \mathcal{M}) \triangleq \inf _{y \in \mathcal{M}}\|z(k)-y\|
$$

which is the smallest distance from $z(k)$ to any point in $\mathcal{M}$. 
To avoid notational confusion, let $x(k) \triangleq$ $\left[x_{1}^{T}(k), \ldots, x_{n}^{T}(k)\right]^{T}$ denote the stacked vector of all deterministic states $x_{i}(k), v_{i} \in \mathcal{V}$, and $\mathbf{X}(k) \triangleq\left[\mathbf{X}_{1}^{T}(k), \ldots, \mathbf{X}_{n}^{T}(k)\right]^{T}$ denote the random variables that represent the random states of $v_{i} \in \mathcal{V}$ at time $k$. Let $x^{F}(k)$ and $x^{L}(k)$ denote the followers' states $x_{i}(k), v_{i} \in \mathcal{V}_{F}$, and the leaders' states $x_{i}(k)$, $v_{i} \in \mathcal{V}_{L}$, respectively. The convex hull spanned by the states of leaders, and all states (i.e., both leaders and followers), are then represented as $\mathrm{Co}\left(x^{L}(k)\right)$ and Co $(x(k))$, respectively. Since the leaders' states are static, the convex hull Co $\left(x^{L}(k)\right)$ is constant, while the convex hull $\mathrm{Co}(x(k))$ is time varying and depends on the states of the followers. The objective is to regulate the states of followers to a desired region, which is a convex hull spanned by all stationary leaders' states (i.e., $\left.x_{i}(k) \rightarrow \operatorname{Co}\left(x^{L}(k)\right)\right)$, over a random communication network.

\section{Consensus algorithms}

Consider the random graph $\mathcal{G}(k)=(\mathcal{V}, \mathcal{E}(k))$ at $t_{k}$. The follower updates its state $\mathbf{X}_{i}(k), v_{i} \in \mathcal{V}_{F}$, according to

$$
\begin{aligned}
\mathbf{X}_{i}(k+1)= & \mathbf{X}_{i}(k) \\
& -\sum_{v_{j} \in \mathcal{N}_{i}(k)} K_{g} \Delta_{t} a_{i j}(k)\left(\mathbf{X}_{i}(k)-\mathbf{X}_{j}(k)\right),
\end{aligned}
$$

where $a_{i j}(k)$ is a random variable defined in (1), $K_{g} \in$ $\mathbb{R}^{+}$is a control gain, $\Delta_{t}$ is a small sampling time, and the time-varying set $\mathcal{N}_{i}(k) \triangleq\left\{v_{j} \mid\left(v_{j}, v_{i}\right) \in \mathcal{E}(k)\right\}$ determines the set of the neighbors of $v_{i}$ in $\mathcal{G}(k)$. Since the leaders are assumed to have desired constant states,

$$
x_{i}(k+1)=x_{i}(k),
$$

for $\forall v_{i} \in \mathcal{V}_{L}$.

Note that $\mathbf{X}_{i}(k)$ is a random variable that evolves according to the stochastic system of (3). Let $\left\{\mathbf{X}_{i}(k)\right\}$ be a Markov process. Since the systems in (3) and (4) along different dimensions are decoupled, for the simplicity of presentation, $\mathbf{X}_{i}(k)$ will be treated as a scalar $(d=1)$ in the subsequent analysis, and the extension of $\mathbf{x}_{i}(k)$ to $d$ dimensional states can be established by using the Kronecker product. The system of (3) and (4) can be rewritten in a compact form as

$$
\left[\begin{array}{c}
x^{L}(k+1) \\
\mathbf{X}^{F}(k+1)
\end{array}\right] \triangleq \Phi(k)\left[\begin{array}{c}
x^{L}(k) \\
\mathbf{X}^{F}(k)
\end{array}\right]
$$

where $\Phi(k) \triangleq\left[\begin{array}{l}\Xi(k) \\ \pi(k)\end{array}\right], \Xi(k) \triangleq\left[\begin{array}{ll}\mathbf{I}_{m \times m} & \mathbf{0}_{m \times(n-m)}\end{array}\right] \in$ $\mathbb{R}^{m \times n}, \mathbf{I}_{m \times m} \in \mathbb{R}^{m \times m}$ denotes an identity matrix, and the entries of $\pi(k) \in \mathbb{R}^{(n-m) \times n}$ are defined as

$$
\pi_{i l}(k)=\left\{\begin{array}{cc}
1-\sum_{v_{j} \in \mathcal{N}_{i}(k)} K_{g} \Delta_{t} a_{i j}(k) & i=l \\
\sum_{v_{j} \in \mathcal{N}_{i}(k)} K_{g} \Delta_{t} a_{i j}(k) & v_{l} \in \mathcal{N}_{i}, i \neq l \\
0, & v_{l} \notin \mathcal{N}_{i}(k), i \neq l .
\end{array}\right.
$$

\section{Convergence Analysis}

In this section, almost sure convergence of the followers' states to the convex hull Co $\left(x^{L}\right)$ spanned by the leaders' states is established for the agreement protocol in (5) over the directed random graph $\mathcal{G}$. To facilitate the subsequent convergence analysis, the definitions of almost sure convergence and supermartingale in a probabilistic setting are introduced.

Definition 3 [17, Ch. 12] A random sequence $\{\mathbf{Z}(k)\}$ in $\mathbb{R}^{n}$ almost surely converges to a set $M$, if for every $\epsilon>0$

$$
\lim _{k_{0} \rightarrow \infty} \operatorname{Pr}\left\{\sup _{k \geq k_{0}} \operatorname{dist}(\mathbf{Z}(k), \mathcal{M})>\epsilon\right\}=0
$$

where dist $(\mathbf{Z}(k), \mathcal{M})$ is defined in (2) representing the distance of $\mathbf{Z}(k)$ to the set $\mathcal{M}$. Almost sure convergence is also called convergence with probability one (w.p.1).

Definition 4 [27] Let $(\Omega, \mathcal{F}, \mathbb{P})$ be a measurable space. A fltration $\mathcal{F}_{0} \subseteq \mathcal{F}_{1} \subseteq \mathcal{F}_{2} \ldots \subseteq \mathcal{F}_{n}$ is an increasing subsequence of sub- $\sigma$-algebras of $\mathcal{F}$. A sequence of random variables $\mathbf{Z}(k)$ is adapted to a filtration $\mathcal{F}_{k}$ if $\mathbf{Z}(k)$ is $\mathcal{F}_{k}$-measurable for all $k$. The pair $(\mathbf{Z}, \mathcal{F})$ is called a supermartingale if, for all $k \geq 0$,

$$
\mathbb{E}[\mathbf{Z}(k)]<\infty \text { and } \mathbb{E}\left[\mathbf{Z}(k+1) \mid \mathcal{F}_{k}\right] \leq \mathbf{Z}(k),
$$

where $\mathbb{E}[\mathbf{Z}(k)]$ denotes the expected value of the random variable $\mathbf{Z}(k)$, and $\mathbb{E}[\square \mid \diamond]$ denotes the conditional expectation of some $\square$ under the condition of some $\diamond$.

The supermartingale sequence $\{\mathbf{Z}(k)\}$ in (8) indicates that the current $\mathbf{Z}(k)$ provides an upper bound for the conditional expectation $\mathbb{E}\left[\mathbf{Z}(k+1) \mid \mathcal{F}_{k}\right]$ on the next time instant $k+1$, and $\lim _{k \rightarrow \infty} \mathbf{Z}(k)$ exists and is finite w. p. 1. In addition, if the sequence $\{\mathbf{Z}(k)\}$ is a nonnegative supermartingale with $\mathbb{E}[\mathbf{Z}(k)]<\infty$ in $(8), \mathbf{Z}(k)$ converges w. p. 1. to a limit [25, Ch. 8].

\subsection{Almost Sure Convergence}

In contrast to most results developed for the deterministic containment control (cf. [8-15]) where network connectivity is the key assumption to ensure consensus (e.g., assuming a tree graph in directed or undirected graphs, 
or at least assuming a tree graph in the union of graphs for switching networks), the random setting in the current work does not make an explicit assumption on network connectivity at each time. The random graph $\mathcal{G}(k)$ can be either connected or disconnected at each time $t_{k}$. However, below we show that Assumptions 2 and 3 ensure that the followers have sufficient access to the leaders' information so that the followers' states will almost surely converge to the convex hull spanned by the leaders' states.

Let $V(x(k)) \in \mathbb{R}^{+}$be the volume of the convex hull Co $(x(k))$ spanned by all leaders' and followers' states at $t_{k}$. The strictly decreasing property of $V(x(k))$ over a finite step is proven in Lemma 1 for the deterministic case of graph $\mathcal{G}_{T}$. For the stochastic case that every edge $\left(v_{j}, v_{i}\right) \in \mathcal{E}$ in $\mathcal{G}$ connects with a probability $p_{i j}>0$, Lemma 2 indicates that $V(\mathbf{X}(k))$ is nonincreasing at each time step. Based on Lemma 1 and Lemma 2, almost sure convergence to the convex hull Co $\left(x^{L}\right)$ for the stochastic system in (3) and (4) is then proven by using convex properties [24] and the stochastic version of LaSalle's invariance principle in [25, Ch. 8].

Lemma 1 Consider the particular directed graph $\mathcal{G}_{T}$ where every potential edge in the random graph $\mathcal{G}=$ $(\mathcal{V}, \mathcal{E})$ is connected. Suppose that Assumption 3 is satisfied, and the followers evolve according to (3), where the control gain $K_{g}$ is selected sufficiently small such that $\sum_{j \in \mathcal{N}_{i}} K_{g} \Delta_{t} a_{i j}(k)<1$. If there exists at least one node $v_{i} \in \mathcal{V}_{F}$ with $x_{i}(k) \notin \mathrm{Co}\left(x^{L}\right)$, the volume of the convex hull $\mathrm{Co}(x(k))$ will strictly decrease over $n_{f}$ steps (i.e., $\mathrm{Co}\left(x\left(k+n_{f}\right)\right) \subset \mathrm{Co}(x(k))$ where $n_{f}$ is the number of followers).

PROOF. Since every potential edge exists in $\mathcal{G}_{T}$, the stochastic dynamics in (3) can be rewritten in a deterministic manner as

$$
\begin{aligned}
x_{i}(k+1)= & \left(1-\sum_{v_{j} \in \mathcal{N}_{i}} K_{g} \Delta_{t} a_{i j}(k)\right) x_{i}(k) \\
& +\sum_{v_{j} \in \mathcal{N}_{i}} K_{g} \Delta_{t} a_{i j}(k) x_{j}(k),
\end{aligned}
$$

for $v_{i} \in \mathcal{V}_{F}$. Here, the random set $\mathcal{N}_{i}(k)$ in $(3)$ is replaced by a constant set $\mathcal{N}_{i}$ determined by $\mathcal{G}_{T}$. Since $K_{g}$ is selected sufficiently small by assumption that $\sum_{j \in \mathcal{N}_{i}} K_{g} \Delta_{t} a_{i j}(k)<1, x_{i}(k+1)$ is a convex combination of $\left\{x_{j}(k)\right\}, v_{j} \in v_{i} \cup \mathcal{N}_{i}$. Note that the convex combination in (9) indicates that each vertex of $\mathrm{Co}(x(k))$ at $k+1$ can either remain in the same state or evolve to shrink Co $(x(k))$ by moving into the interior or along the boundary of $\mathrm{Co}(x(k))$, resulting in $\mathrm{Co}(x(k+1)) \subseteq \mathrm{Co}(x(k))$. To show that the equality can be excluded in this set relation over $n_{f}$ steps (i.e.,
$\left.\mathrm{Co}\left(x\left(k+n_{f}\right)\right) \subset \mathrm{Co}(x(k))\right)$, we will prove that at least one vertex of Co $(x(k))$ evolves to shrink Co $(x(k))$ over $n_{f}$ steps. Since the existence of a node $v_{i} \in \mathcal{V}_{F}$ with $x_{i}(k) \notin \mathrm{Co}\left(x^{L}\right)$ ensures that $\mathrm{Co}\left(x^{L}\right) \subset \mathrm{Co}(x(k))$, there always exists a vertex of $\mathrm{Co}(x(k))$ formed by followers only. Two cases for the component of such vertex are considered.

Case 1: Consider a vertex of $\operatorname{Co}(x(k))$ consisting of a single follower $v_{i} \in \mathcal{V}_{F}$ such that $x_{i}(k) \notin \operatorname{Co}\left(x^{L}\right)$. Since $\mathcal{G}_{T}$ contains a directed path from $\mathcal{V}_{L}$ to every node in $\mathcal{V}_{F}$, there exists at least one node $v_{j} \in \mathcal{N}_{i}$ either in the interior or on the boundary of $\operatorname{Co}(x(k))$ with a different state $x_{j}(k) \neq x_{i}(k)$. According to $(3)$, the vertex state $x_{i}(k+1)$ will evolve either along the boundary or to the interior of $\mathrm{Co}(x(k))$ to shrink $\mathrm{Co}(x(k))$, resulting in $\mathrm{Co}(x(k+1)) \subset \operatorname{Co}(x(k))$.

Case 2: Consider a vertex of $\mathrm{Co}(x(k))$ consisting of multiple followers $\left\{v_{i} \in \mathcal{V}_{F}\right\}$ with the same states and each $x_{i}(k) \notin$ Co $\left(x^{L}\right)$. Let $S_{v}$ be the set of followers that forms such vertex. The worst case is that $S_{v}$ contains all followers $v_{i}, i \in\left\{1, \ldots, n_{f}\right\}$, with $x_{1}(k)=\ldots=x_{n_{f}}(k)$. According to (3), for those nodes $v_{i}$ with $\mathcal{N}_{i} \subset S_{v}$, $x_{i}(k+1)=x_{i}(k)$, due to $x_{i}(k)=x_{j}(k), v_{j} \in \mathcal{N}_{i}$. However, $\mathcal{G}_{T}$ ensures a directed path from $\mathcal{V}_{L}$ to at least one node $v_{j} \in S_{v}$ with $v_{k} \in \mathcal{N}_{j}$ and $v_{k} \notin S_{v}$. Following (3), $x_{j}(k+1)$ moves out of the vertex either into the interior or along the boundary of $\mathrm{Co}(x(k))$. Due to the connected $\mathcal{G}_{T}$, there always exists a node $v_{j}$ in $S_{v}$ with at least one neighbor $v_{k} \in \mathcal{N}_{j}$ and $v_{k} \notin S_{v}$. Repeating the above process, the number of followers in $S_{v}$ is strictly decreasing at each update, and Case 2 will reduce to Case 1 with the vertex containing a single follower for at most $n_{f}$ steps, resulting in $\mathrm{Co}\left(x\left(k+n_{f}\right)\right) \subset \mathrm{Co}(x(k))$ based on Case 1.

Lemma 2 Let $\mathcal{Q}_{\lambda}=\left\{x \in \mathbb{R}^{n} \mid V(x) \leq \lambda\right\}$ where $\lambda>0$. Given that the sequence $\{\mathbf{X}(k)\}$ evolves according to the dynamics in (3) and (4), if $x(0)$ starts in $\mathcal{Q}_{\lambda}, \mathbf{X}(k) \in \mathcal{Q}_{\lambda}$ for all $k \in \mathbb{Z}^{+}$w. p. 1., that is,

$$
\operatorname{Pr}\left(\sup _{0<k<\infty} V(\mathbf{X}(k))>\lambda\right)=0 .
$$

PROOF. To show (10), it suffices to show that $V(\mathbf{X}(k+1)) \leq V(\mathbf{X}(k))$. Let $\overline{\mathcal{N}}_{i}(k)$ denote the set of all potential neighbors of $v_{i}$ on $\mathcal{G}_{k}$. Consider a follower node $v_{i} \in \mathcal{V}_{F}$ with a neighbor set $\mathcal{N}_{i}(k)$ on $\mathcal{G}_{k}$, where $\mathcal{N}_{i}(k) \subseteq \overline{\mathcal{N}}_{i}(k)$ indicates the set of nodes that are connected to $v_{i}$ at $t_{k}$. Since each edge $\left(v_{j}, v_{i}\right) \in \mathcal{E}_{k}$, $v_{j} \in \mathcal{N}_{i}(k)$, is connected with a probability $p_{i j}$, the node $v_{i}$ may connect to either a subset of nodes in $\overline{\mathcal{N}}_{i}$ or none of the nodes in $\overline{\mathcal{N}}_{i}$. If $v_{i}$ connects to at least one node in $\mathcal{N}_{i}(k)$, according to Lemma $1, x_{i}(k)$ can either remain the same state or evolve to shrink the convex hull formed 
by itself and its neighbors $v_{j} \in \mathcal{N}_{i}(k)$, resulting in a nonincreasing $V\left(x_{k}\right)$. If $v_{i}$ is isolated, which indicates that no edge exists between $v_{i}$ and any other node in $\mathcal{N}_{i}(k)$, $x_{i}(k)$ will remain the same and not lead to an increasing volume of the convex hull $\mathrm{Co}(x(k))$. Repeating this argument for every node in the graph $\mathcal{G}_{k}$ indicates that $V(\mathbf{X}(k+1)) \leq V(\mathbf{X}(k))$.

Theorem 1 Consider the random graph $\mathcal{G}=(\mathcal{V}, \mathcal{E})$ that consists of stationary leaders and dynamic followers described by the stochastic system in (3) and (4). If Assumption 3 is satisfied, the followers $v_{i} \in V_{F}$ will almost surely converge to the convex hull $\mathrm{Co}\left(x^{L}\right)$ that is spanned by the leaders states only.

PROOF. The theorem is proven by using the stochastic version of LaSalle's invariance principle in [25, Ch. $8]$ and convex properties in [24, Ch. 2]. Consider a time sequence $T_{m}, m \in \mathbb{Z}^{+}$, with $T_{m+1}-T_{m}=n_{f} \Delta_{t}$ and $T_{0}=t_{0}$. First, we show that there exists a positively invariant set $\mathcal{Q}$ such that if $x(0) \in \mathcal{Q}$, then $\mathbf{X}\left(T_{m}\right) \in \mathcal{Q}$ for all $T_{m} \geq 0$. Second, the volume of the convex hull $\mathrm{Co}(x(0))$ is decreasing to the invariant set $E \subseteq \mathcal{Q}$ where the volume of $\mathrm{Co}\left(\mathbf{X}\left(T_{m}\right)\right), \mathbf{X}\left(T_{m}\right) \in E$, stays constant. It is then shown that $M$ is the largest invariant set in $E$, where $M$ is the set of points in the convex hull Co $\left(x^{L}\right)$ formed by stationary leaders only.

Let $\mathcal{Q} \triangleq \operatorname{Co}(x(0))$, where $\mathrm{Co}(x(0))$ is the convex hull formed by all initial states $x(0)$. Since Lemma 2 indicates that $\mathbf{X}(k) \in \mathcal{Q}$ for all $k \in \mathbb{Z}^{+}$w. p. 1. if $x(0)$ starts in $\mathcal{Q}$, $\mathbf{X}\left(T_{m}\right) \in \mathcal{Q}$ also holds for all $m \in \mathbb{Z}^{+}$w. p. 1., indicating that $\mathcal{Q}$ is indeed a positively invariant set. To show that all the followers almost surely converge to the convex hull Co $\left(x^{L}\right)$, consider the auxiliary term $\delta\left(x\left(T_{m}\right)\right) \in \mathbb{R}$ defined as

$$
\begin{aligned}
\delta\left(x\left(T_{m}\right)\right) \triangleq & V\left(x\left(T_{m}\right)\right) \\
& -\mathbb{E}\left[V\left(\mathbf{X}\left(T_{m+1}\right)\right) \mid \mathbf{X}\left(T_{m}\right)=x\left(T_{m}\right)\right] .
\end{aligned}
$$

In (11), the state $x\left(T_{m}\right)$ evolves according to (5) by following a sequence of $n_{f}$ random graphs, where, by Assumption 1, each random graph stays constant for a period of $\Delta_{t}$ over the interval $\left[T_{m}, T_{m+1}\right]=n_{f} \Delta_{t}$. To capture all possible sequences of the evolution from $x\left(T_{m}\right)$ to $x\left(T_{m+1}\right)$, let $\overline{\mathcal{G}}^{\prime}=\left\{\mathcal{G}_{1}^{\prime}, \ldots, \mathcal{G}_{M_{T}}^{\prime}\right\}$ denote the finite set of all possible sequences over $\left[T_{m}, T_{m+1}\right]$, where $M_{T} \in \mathbb{Z}^{+}$denotes the cardinality of $\overline{\mathcal{G}}^{\prime}$ (i.e., $M_{T}=\left|\overline{\mathcal{G}}^{\prime}\right|$ ) and each entry $\mathcal{G}_{i}^{\prime} \in \overline{\mathcal{G}}^{\prime}, i \in\left\{1, \ldots, M_{T}\right\}$, denotes a possible sequence that contains $n_{f}$ graphs (i.e., $\mathcal{G}_{i}^{\prime} \in$ $\left.\prod_{i=1}^{n_{f}} \overline{\mathcal{G}}\right)$. Let $p_{i}^{\prime} \triangleq \operatorname{Pr}\left(\mathcal{G}_{i}^{\prime} \in \overline{\mathcal{G}}^{\prime}\right)$ be the probability of the occurrence of the sequence $\mathcal{G}_{i}^{\prime}$ in $\overline{\mathcal{G}}^{\prime}$.

Given the definition of the $\overline{\mathcal{G}}^{\prime}$ and the associated probability for each entry in $\overline{\mathcal{G}}^{\prime}$, the conditional expectation in (11) is computed as

$$
\begin{array}{r}
\mathbb{E}\left[V\left(\mathbf{X}\left(T_{m+1}\right)\right) \mid \mathbf{X}\left(T_{m}\right)=x\left(T_{m}\right)\right] \\
=\sum_{j=1}^{M_{T}} V\left(\Phi_{j}^{\prime} x\left(T_{m}\right)\right) p_{j}^{\prime}
\end{array}
$$

where $\Phi_{j}^{\prime}=\prod_{i=1}^{n_{f}} \Phi_{i}$ corresponds to the combined state transition matrix associated with the sequence $\mathcal{G}_{j}^{\prime}$ in $\overline{\mathcal{G}}^{\prime}$, and each $\Phi_{i}$ is the corresponding state transition matrix in (5) for the random graph at $\left[t_{n_{f} m}, t_{n_{f} m}+i \Delta_{t}\right]$. Let $\mathcal{G}_{T}^{\prime}$ be a path in $\overline{\mathcal{G}}^{\prime}$ that consists of $\mathcal{G}_{T}$ only. By Assumption 2, the probability $p_{T}^{\prime}$ that $\mathcal{G}_{T}$ occurs consecutively $n_{f}$ times in $\mathcal{G}_{T}^{\prime}$ during $\left[T_{m}, T_{m+1}\right]$ is strictly greater than zero, $p_{T}^{\prime}>0$. The strict decreasing property of $V\left(\Phi_{T}^{\prime} x\left(T_{m}\right)\right) p_{T}^{\prime}$ is then established by Assumption 2 and Lemma 1, where $\Phi_{T}^{\prime}$ indicates the state transition matrix corresponding to $\mathcal{G}_{T}^{\prime} \in \overline{\mathcal{G}}^{\prime}$. For those entries $\mathcal{G}_{j}^{\prime} \in \overline{\mathcal{G}}^{\prime}$ other than $\mathcal{G}_{T}^{\prime}$, the convex properties ensure that each $V\left(\Phi_{j}^{\prime} x\left(T_{m}\right)\right), j \in\left\{1, \ldots, M_{T}\right\}$ and $j \neq T$ in (12) is a nonincreasing function as shown in Lemma 2. Hence, when considering all possible graphs in $\overline{\mathcal{G}}$,

$$
\mathbb{E}\left[V\left(\mathbf{X}\left(T_{m+1}\right)\right) \mid \mathbf{X}\left(T_{m}\right)=x\left(T_{m}\right)\right]<V\left(x\left(T_{m}\right)\right),
$$

which indicates that the sequence of $\left\{V\left(\mathbf{X}\left(T_{m}\right)\right)\right\}$ is a supermartingale. Since $\left\{V\left(\mathbf{X}\left(T_{m}\right)\right)\right\}$ is a nonnegative supermartingale and bounded in $\mathcal{Q}$, based on Definition 4 and Theorem 1 in $\left[25\right.$, Ch. 8], $V\left(\mathbf{X}\left(T_{m}\right)\right)$ will decrease to a limit, which indicates that the convex hull Co $(\mathbf{X}(k))$ will shrink to an invariant set as $m \rightarrow \infty$.

Let $E$ be the invariant set of all points in $\mathcal{Q}$, where the volume of $\mathrm{Co}\left(\mathbf{X}\left(T_{m}\right)\right), \mathbf{X}\left(T_{m}\right) \in E$, stays constant. Based on LaSalle's Theorem, the largest invariant set $M$ must be established, where $M$ is the set of points in the convex hull Co $\left(x^{L}\right)$ formed by stationary leaders only. A proof by contradiction is used to show that $M$ (i.e., $\left.\mathrm{Co}\left(x^{L}\right)\right)$ is the largest invariant set. Let $M^{\prime} \supset M$ be a larger invariant set in $E$. Suppose that there is a follower whose state $x_{i} \notin M$, and $x_{i}$ is on the boundary of $M^{\prime}$, while the other followers $x_{j} \in M, v_{j} \in \mathcal{V}_{F}-\left\{v_{i}\right\}$. Since $M^{\prime} \subset E$, the volume of the set $M^{\prime}$ stays constant. The only way for the volume of $M^{\prime}$ to stay constant is that $x_{i}(t)$ stays constant for all $t \geq 0$. However, for this to happen, the follower $v_{i}$ must be isolated from the group for all $t \geq 0$. This isolation is a contradiction with Assumption 2. Hence, $M$ is the largest invariant set. A stochastic version of LaSalle's invariance principle in [25, Ch. 8] can now be invoked to ensure almost sure convergence of the followers' states to the largest invariant set $M$ (i.e., the convex hull Co $\left(x^{L}\right)$ ).

Remark 1 Containment control for a multi-agent system has received significant focus (cf. [8-16]). How- 
ever, few results investigate collision avoidance among agents in containment control over either deterministic or stochastic communication networks. In our previous work [14], containment control is applied to a deterministic social network to regulate the emotional states of individuals to a desired end. To maintain the social bond within a desired threshold (i.e., $\left\|q_{i}(t)-q_{j}(t)\right\| \leq \delta$, where $q_{i}$ and $q_{j}$ denote the time-varying social states of individual $i$ and $j$, respectively, and $\delta \in \mathbb{R}^{+}$denotes the threshold), a navigation function based framework from [28] is developed to ensure existing social influence between individuals during network evolution. Since ensuring collision avoidance among agents (i.e., $\left\|x_{i}(t)-x_{j}(t)\right\| \geq \xi$, where $x_{i}$ and $x_{j}$ denote the timevarying positions of agent $i$ and $j$, respectively, and $\xi \in \mathbb{R}^{+}$denotes a safe inter-agent distance) is analogous to maintaining existing social influence between individuals, the approach developed in [14] could be modified to handle collision avoidance among agents in containment control. In another recent work [29], a navigation function based distributed controller is developed for a multiagent system to perform formation control with ensured collision avoidance over an intermittent sensing network where the agents suffer random loss of inter-agent sensing and communication. Based on the stochastic analysis framework developed in the present work, the approach developed in [29] could also be extended for collision avoidance in containment control over stochastic communication networks.

\section{Simulation}

A graph of 100 nodes in a two-dimensional coordinate space is considered, where the states of nodes are their locations in the two-dimensional coordinate space. The followers and the leaders form a directed graph, represented by the dots and squares in Fig. 1, respectively. To show random loss of the communication links, a twostate Markov Model with independent edges is applied to represent the evolution of the edge states. Particularly, each edge $(i, j)$ will change its current state (i.e., from connection to disconnection or from disconnection to connection) with probability $p_{i j}$ and will remain the current state (i.e., connection or disconnection) with probability $1-p_{i j}$. Consider the case that $p_{i j} \in(0,1)$ is randomly generated for each edge in the graph. Fig. 1 indicates that the followers converge to the locations inside the convex hull spanned by the stationary leaders on the given random graph. We also compared the performance for the same graph with different connection models and the results are shown in Fig. 2. In the comparison, all graphs start from the same initial graph and differ in how the edge connections are evolved according to the twostate Markov Model. The solid line in Fig. 2 indicates the evolution of $\mathrm{Co}(x)$ for a fixed connection model where all potential edges are connected all the time. The dashed and dot-dashed lines in Fig. 2 represent the evolution of $\mathrm{Co}(x)$ for the Markov Model with independent edges

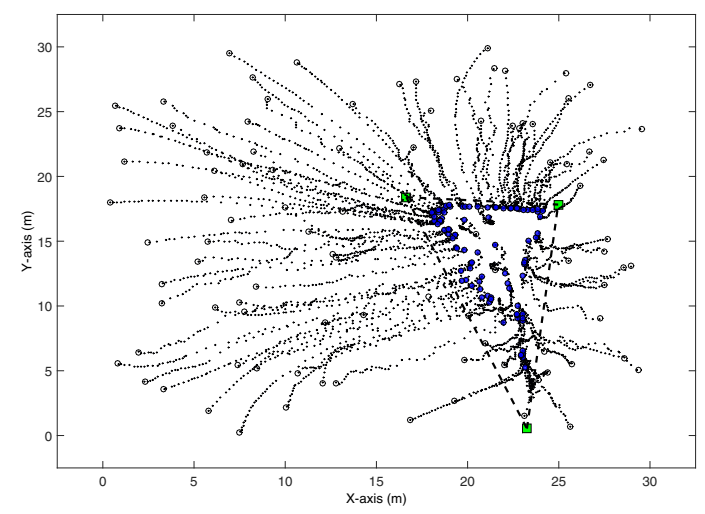

Figure 1. Node trajectories plot. The followers' states converge to the convex hull formed by stationary leaders, where the the leaders' states are denoted as squares, and the followers' initial and final states are denoted by circles and dots, respectively.

that each edge $(i, j)$ fails or succeeds with high state transition probability $p_{i j} \in[0.95,1)$ and low state transition probability $p_{i j} \in(0,0.05]$, respectively. The dot line shows a Markov Model with dependent edges that all fail or succeed at the same time, where the stationary state probabilities are generated randomly from a uniform $(0,1)$ distribution. As expected, the performance for the fixed graph outperforms other connection models, since constant information exchange is available all the time. However, such a fully connected graph appears rarely in a random network that evolves according to the two-state Markov Model (e.g., appearing only 4 times for a simulation of 15,000 times). Note that the algorithm developed in the current work can achieve the same containment result without requiring the graph to be regularly fully connected, as shown in Fig. 2. The graph with dependent edges and the graph with high state transition probability behaves similar to each other, converging to the convex hull Co $\left(x^{L}\right)$ at about the same speed. The graph with low state transition probability takes the longest time to converge, since the graph may run into a disconnected graph and stay there for a relatively long time due to its low probability in state transition, as shown by the non-strictly decreasing dot-dashed line.

\section{Conclusion}

Leader-follower containment control on random graphs is examined. The underlying random network is assumed to evolve according to a two-state Markov Model, and the followers are proven to converge to the convex hull spanned by the stationary leaders w. p. 1. The simulation results demonstrate the convergence of all followers to the convex hull Co $\left(x^{L}\right)$ over random networks. Although convergence on random graphs is established, convergence speed is not investigated in the current work. Since the convergence speed of consensus highly 


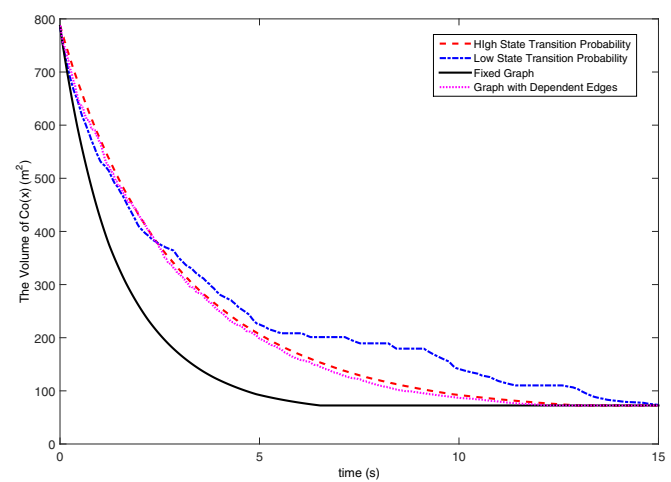

Figure 2. The evolution of the volume of the convex hull Co $(x)$ for the graph with different connection models.

relies on the topology of the underlying communication network, additional work will examine the topology design to increase the convergence speed for the developed leader-follower containment control on random graphs.

\section{References}

[1] W. Ren, R. W. Beard, and E. M. Atkins, "Information consensus in multivehicle cooperative control," IEEE Control Syst. Mag., vol. 27, pp. 71-82, April 2007.

[2] R. Olfati-Saber, J. A. Fax, and R. M. Murray, "Consensus and cooperation in networked multi-agent systems," Proc. IEEE, vol. 95, no. 1, pp. 215 - 233, Jan. 2007.

[3] H. G. Tanner, A. Jadbabaie, and G. J. Pappas, "Flocking in fixed and switching networks," IEEE Trans. Autom. Control, vol. 52, no. 5, pp. 863-868, May 2007.

[4] A. Jadbabaie, J. Lin, and A. Morse, "Coordination of groups of mobile autonomous agents using nearest neighbor rules," IEEE Trans. Autom. Control, vol. 48, no. 6, pp. 988-1001, June 2003.

[5] M. Zhu and S. Martínez, "Discrete-time dynamic average consensus," Automatica, vol. 46, no. 2, pp. 322-329, 2010.

[6] D. V. Dimarogonas and K. J. Kyriakopoulos, "On the rendezvous problem for multiple nonholonomic agents," IEEE Trans. Autom. Control, vol. 52, no. 5, pp. 916-922, May 2007.

[7] Z. Kan, L. Navaravong, J. Shea, E. Pasiliao, and W. E. Dixon, "Graph matching based formation reconfiguration of networked agents with connectivity maintenance," IEEE Trans. Control Netw. Syst., vol. 2, no. 1, pp. 24-35, March 2015.

[8] M. Ji, G. Ferrari-Trecate, M. Egerstedt, and A. Buffa, "Containment control in mobile networks," IEEE Trans. Autom. Control, vol. 53, no. 8, pp. 1972-1975, 2008.

[9] Z. Meng, W. Ren, and Z. You, "Distributed finite-time attitude containment control for multiple rigid bodies," Automatica, vol. 46, no. 12, pp. 2092-2099, 2010.

[10] Y. Cao, W. Ren, and M. Egerstedt, "Distributed containment control with multiple stationary or dynamic leaders in fixed and switching directed networks," Automatica, vol. 48, pp. 1586-1597, 2012.

[11] J. Li, W. Ren, and S. Xu, "Distributed containment control with multiple dynamic leaders for double-integrator dynamics using only position measurements," IEEE Trans. Autom. Control, vol. 57, no. 6, pp. 1553-1559, 2012.

[12] G. Notarstefano, M. Egerstedt, and M. Haque, "Containment in leader-follower networks with switching communication topologies," Automatica, vol. 47, no. 5, pp. 1035-1040, 2011.

[13] Y. Lou and Y. Hong, "Target containment control of multi-agent systems with random switching interconnection topologies," Automatica, vol. 48, no. 5, pp. 879-885, 2012.

[14] Z. Kan, J. Klotz, E. L. P. Jr, and W. E. Dixon, "Containment control for a social network with statedependent connectivity," Automatica, vol. 56, pp. 86-92, June 2015 .

[15] J. Mei, W. Ren, and G. Ma, "Distributed containment control for Lagrangian networks with parametric uncertainties under a directed graph," Automatica, vol. 48, no. 4, pp. 653-659, 2012.

[16] Y. Wang, L. Cheng, Z.-G. Hou, M. Tan, and M. Wang, "Containment control of multi-agent systems in a noisy communication environment," Automatica, vol. 50, no. 7, pp. 1922-1928, 2014.

[17] Y. Hatano and M. Mesbahi, "Agreement over random networks," IEEE Trans. Autom. Control, vol. 50, no. 11, pp. 1867-1872, 2005.

[18] C. W. Wu, "Synchronization and convergence of linear dynamics in random directed networks," IEEE Trans. Autom. Control, vol. 51, no. 7, pp. 1207-1210, 2006.

[19] M. Porfiri and D. J. Stilwell, "Consensus seeking over random weighted directed graphs," IEEE Trans. Autom. Control, vol. 52 , no. 9 , pp. 1767-1773, 2007.

[20] A. Tahbaz-Salehi and A. Jadbabaie, "A necessary and sufficient condition for consensus over random networks," IEEE Trans. Autom. Control, vol. 53, no. 3, pp. 791-795, 2008.

[21] Y. Zhang and Y.-P. Tian, "Consensus of data-sampled multiagent systems with random communication delay and packet loss," IEEE Trans. Autom. Control, vol. 55, no. 4, pp. 939943, 2010.

[22] — - "Maximum allowable loss probability for consensus of multi-agent systems over random weighted lossy networks," IEEE Trans. Autom. Control, vol. 57, no. 8, pp. 2127-2132, 2012.

[23] Y. Wang, L. Cheng, W. Ren, Z.-G. Hou, and M. Tan, "Seeking consensus in networks of linear agents: communication noises and markovian switching topologies," IEEE Trans. Autom. Control, vol. 60, no. 5, pp. 1374-1379, 2015.

[24] S. Boyd and L. Vandenberghe, Convex Optimization. New York, NY, USA: Cambridge University Press, 2004.

[25] H. J. Kushner, Introduction to Stochastic Control. New York: Holt, Reinhart and Winston, 1971.

[26] H. K. Khalil, Nonlinear Systems, 3rd ed. Upper Saddle River, NJ, USA: Prentice Hall, 2002.

[27] G. Grimmett and D. Stirzaker, Probability and random processes. Oxford University Press, 2001.

[28] Z. Kan, A. Dani, J. M. Shea, and W. E. Dixon, "Network connectivity preserving formation stabilization and obstacle avoidance via a decentralized controller," IEEE Trans. Autom. Control, vol. 57, no. 7, pp. 1827-1832, 2012.

[29] T.-H. Cheng, Z. Kan, J. A. Rosenfeld, A. Parikh, and W. E. Dixon, "Decentralized formation control with connectivity maintenance and collision avoidance under limited and intermittent sensing," in Proc. Am. Control Conf., Portland, Oregon, USA, June 2014, pp. 3201-3206. 